\title{
Escala de Positividade: Evidências Iniciais de Validade para Adolescentes Brasileiros ${ }^{1}$
}

\author{
Juliane Callegaro Borsa², Andrea Jannotti Nogueira Rodrigues, Bheatrix Bienemann \\ Pontifícia Universidade Católica do Rio de Janeiro, Rio de Janeiro-RJ, Brasil \\ Bruno Figueiredo Damásio \\ Universidade Federal do Rio de Janeiro, Rio de Janeiro-RJ, Brasil
}

\section{RESUMO}

A positividade refere-se à disposição do indivíduo para avaliar positivamente os diversos aspectos da vida. Estudos demonstram os efeitos benéficos da positividade na vida das pessoas em diversos domínios, incluindo a performance no trabalho e nas relações sociais. $\mathrm{Na}$ adolescência, a positividade apresenta-se como um importante fator de proteção para o desenvolvimento saudável. O presente estudo apresenta evidências iniciais de validade da Escala de Positividade (EP) para uma amostra de 638 adolescentes brasileiros (67,7 $\%$ do sexo feminino; idade média $=15,19 ; D P=2,40)$. Análises fatoriais exploratória e confirmatória indicaram solução unifatorial para a EP, corroborando sua estrutura original. A validade convergente foi evidenciada por meio de correlações positivas moderadas entre a EP e os indicadores de esperança, otimismo, autoestima e saúde mental. Este estudo amplia as evidências de validade da EP no contexto brasileiro e oferece uma nova ferramenta para os estudos da Psicologia Positiva desenvolvidos com adolescentes.

Palavras-chave: psicologia positiva, adolescentes, positividade, evidência de validade.

\section{ABSTRACT - Positivity Scale: initial validity evidence for Brazilian adolescents}

Positivity refers to an individual's willingness to positively evaluate various aspects of life. Studies demonstrate the beneficial effects of positivity on people's lives in various domains, including work performance and social relationships. In adolescence, positivity is an important protective factor for healthy development. This study presents initial validity evidence of the Positivity Scale (PS) for a sample of 638 Brazilian adolescents $(67.7 \%$ female, mean age $=15.19, S D=2.40)$. Exploratory and confirmatory factor analyses indicated a single factor solution for PS, corroborating its original structure. Convergent validity was evidenced by moderate positive correlations between PS and indicators of hope, optimism, self-esteem, and mental health. This study expands the validity evidence of PS in the Brazilian context and offers a new tool for the studies of Positive Psychology developed with adolescents.

Keywords: Positive Psychology; Adolescents; Positivity; Validity Evidence.

\section{RESUMEN - Escala de Positividad: evidencias iniciales de validez para adolescentes brasileños}

La positividad consiste en la disposición de los individuos para evaluar de forma positiva los diversos aspectos de la vida. Estudios han demostrado los efectos benéficos de la positividad en la vida de las personas en diversos ámbitos, tales como rendimiento en el trabajo y en las relaciones sociales. En la adolescencia, la positividad se presenta como un importante factor de protección para el desarrollo saludable. El presente estudio tuvo como objetivo presentar evidencias iniciales de validez de la Escala de Positividad (EP) en una muestra de 638 adolescentes brasileños $(67,7 \%$ de sexo femenino; edad media $=15,19 ; D P=2,40)$. Los análisis factoriales exploratorios y confirmatorios indicaron solución unifactorial para la EP, corroborando su estructura original. La validez convergente fue evidenciada por medio de correlaciones positivas moderadas entre la EP y los indicadores de esperanza, optimismo, autoestima y salud mental. Este estudio amplía las evidencias de validez de la EP en el contexto brasileño y ofrece una nueva herramienta para los estudios de psicología positiva con adolescentes.

Palabras clave: Psicología Positiva; Adolescentes; Positividad; Evidencia de Validez.

\section{Introdução}

Nas últimas décadas, os aspectos positivos do funcionamento individual vêm recebendo especial atenção de pesquisadores, em conformidade com uma visão de que indivíduos felizes e plenamente realizados tendem a administrar suas vidas de forma bem-sucedida, contribuindo efetivamente à comunidade e às demandas sociais (Csikszentmihalyi, 2014; Seligman \& Csikszentmihalyi, 2014). Autoestima, satisfação com a vida e otimismo são algumas das características comumente investigadas na literatura, já que estão frequentemente associadas

${ }^{1}$ Apoio Financeiro: Capes.

2 Endereço para correspondência: Rua Marquês de São Vicente, 225, Edifício Cardeal Leme, 2 Andar - Sala 201, Gávea, 22451-900, Rio de Janeiro-RJ. Tel.: (21) 3527-2086. Cel.: (21) 96971-9990. E-mail: juliborsa@gmail.com 
a desfechos positivos de vida (Caprara, Eisenberg, \& Alessandri, 2016; Diener, Kanazawa, Suh, \& Oishi, 2015; Lyubomirsky, \& Layous, 2013; Moksnes, \& Espnes, 2013). A tríade composta por essas características é denominada "positividade", conceito desenvolvido por Caprara et al. que se refere à disposição geral do indivíduo para avaliar positivamente os diversos aspectos da vida (Caprara et al., 2012a, 2012b; Diener, Scollon, Oishi, Dzokoto, \& Suh, 2000; Oles et al., 2013).

Estudos tem demonstrado os efeitos benéficos da positividade na vida das pessoas em diversos domínios, tais como saúde física e psicológica, performance no trabalho e nas relações sociais (Alessandri et al., 2012; Caprara, Eisenberg, \& Alessandri, 2016). Nas pesquisas relacionadas à Psicologia Positiva, a positividade vem sendo estudada como um compósito constituído pelo que existe de comum entre as variáveis Satisfação com a Vida, Autoestima e Otimismo (Caprara et al., 2012a). Essas variáveis, por sua vez, estão relacionadas positivamente com afetos positivos, estabilidade emocional; além de se correlacionarem negativamente com depressão, afetos negativos e outros indicadores de psicopatologia (Caprara, Steca, Alessandri, Abela, \& McWhinnie, 2010).

Para investigar a positividade em adultos, Caprara et al., (2012a, 2012b) desenvolveram a Escala de Positividade (EP), a qual tem por objetivo avaliar a visão positiva que as pessoas têm sobre si, sobre a vida e sobre o futuro, e ainda, a confiança em relação às outras pessoas. Trata-se de uma escala de autorrelato do tipo Likert composta por oito itens, cujas respostas podem variar de discordo totalmente a concordo totalmente. A EP tem sido utilizada em diferentes culturas, apresentando invariância estrutural em países como Itália, Canadá, Alemanha e Japão (Caprara et al., 2012a, 2012b).

No Brasil, a EP foi adaptada e validada por Borsa, Damásio, Souza, Koller e Caprara (2015) em uma amostra de 730 indivíduos (65\% mulheres), com idades entre 17 e 70 anos de idade $(M=31,0$ anos, $D P=11,43)$, residentes em 21 estados brasileiros. A análise fatorial exploratória corroborou o estudo original (Caprara et al., 2012a) ao indicar a unidimensionalidade da EP (cargas fatoriais variando de $-0,36$ a 0,87$)$. A análise fatorial confirmatória, por sua vez, apresentou índices de ajuste adequados ao modelo $\left(\chi^{2}(d f)=113,98(20), p<0,001 ;\right.$ SRMR $=0,065$; $\mathrm{CFI}=0,95$; TLI $=0,93$; RMSEA $=0,11$ [09-0,13]). Os resultados indicaram, ainda, correlação positiva entre a EP e as variáveis saúde mental, sentido de vida, felicidade subjetiva e satisfação com a vida. Não foram encontradas diferenças significativas entre sexo $[\mathrm{F}(1,670)=0,215$, $p=0,64]$, paternidade $[\mathrm{F}(1,670)=0,242, \quad p=0,62]$, e religiosidade/espiritualidade [F $(1,670)=0,808, p=0,37]$. Os escores de positividade na amostra brasileira apresentaram baixa correlação positiva com as variáveis idade, escolaridade e renda mensal. Os resultados sugerem que a positividade está mais relacionada às características individuais do que aos aspectos sociodemográficos, corroborando as hipóteses de Caprara et al. (Caprara et al., 2010; Caprara et al., 2012a).

Em estudo posterior, Borsa, Damásio e Koller (2016) investigaram novas evidências de validade para EP em uma amostra de 876 indivíduos brasileiros $(66,0 \%$ mulheres), com idades variando entre 18 e 83 anos $(M=32,09 ; D P=10,98)$. A análise fatorial confirmatória multigrupo indicou invariância configural, métrica e escalar, demostrando que a EP pode ser utilizada indistintamente para homens e mulheres. Análises de regressão linear múltipla foram realizadas para avaliar a relação da EP com variáveis constituintes do construto positividade (modelo 1) e com variáveis convergentes (modelo 2). O primeiro modelo evidenciou que a positividade é, de fato, um construto composto pelas variáveis autoestima, satisfação com a vida e otimismo (Caprara et al., 2010; Caprara, Eisenberg, \& Alessandri, 2016) haja vista que estas explicaram aproximadamente $80 \%$ dos escores da $\mathrm{EP}$, mesmo quando inseridas variáveis correlatas (sentido de vida, vitalidade, felicidade subjetiva, autoeficácia, estresse, depressão e ansiedade), que explicaram apenas $1,8 \%$ dos escores da EP. O Modelo 2, por sua vez, confirmou a relação da positividade com outras variáveis, corroborando diversos estudos conduzidos no âmbito da Psicologia Positiva (e.g., Alessandri, Caprara, \& Tisak, 2012; Alessandri et al., 2012; Costantini et al., 2016). Nesse segundo modelo, o percentual de variância explicada foi de $71,2 \%$, sendo que estas mesmas variáveis, no Modelo 1, explicaram apenas 1,8\% do construto. Essa discrepância mostra o quanto as variáveis constitutivas da positividade são capazes de predizer o construto, enquanto outras variáveis correlatas perdem o seu poder de explicação quando inseridas em um único modelo. Os estudos de Borsa et al. corroboram os estudos originais, sugerindo que a EP é uma medida confiável para avaliar a positividade de adultos brasileiros.

Estudos longitudinais têm demonstrado que a positividade é uma característica relativamente estável e que pouco varia entre a adolescência e a idade adulta (Alessandri, Caprara, \& Tisak, 2012). No entanto, estudos sobre a positividade em adolescentes ainda são negligenciados na literatura.

A adolescência é uma etapa de transição importante, caracterizada pela saída da infância e pelo início da vida adulta e que precisa ser compreendida para além de um mero período de transição, já que representa uma importante etapa do desenvolvimento, sobretudo no que se refere à aquisição de importantes competências cognitivas, afetivas e sociais (Santrock, 2014; Senna \& Dessen, 2012). No entanto, as mudanças biológicas e sociais presentes durante essa fase - caracterizada muitas vezes por frequentes alterações de humor e problemas comportamentais - podem ser desafiadoras e causadoras de considerável estresse nesses indivíduos (Albert, Chein, \& Steinberg, 2013). Um relatório recente da Organização Mundial de Saúde (OMS) indicou, por exemplo, que o 
suicídio é a segunda maior causa de morte de jovens entre 15 e 29 anos (World Health Organization, 2014), o que se configura como mais uma evidência da vulnerabilidade psicológica dessa população.

Conforme aponta Caprara et al. (2006), para que haja o desenvolvimento saudável do adolescente, é necessário mais do que uma perspectiva que visa a correção de deficiências, mas uma perspectiva que considere a construção de competências. Uma visão marcada por um desenvolvimento positivo é essencial para substituir as crenças de que a adolescência é um período conturbado, bem como para prevenir o engajamento dos jovens em comportamentos autodestrutivos e de risco (Albert, Chein, \& Steinberg, 2013; Johnstone, Rooney, Hassan, $\&$ Kane, 2014). Nesse sentido, torna-se importante compreender o impacto das características psicológicas positivas em adolescentes (Caprara et al., 2012a; OrejudoHernández, Aparicio-Moreno, \& Cano-Escoriaza, 2014).

$\mathrm{Na}$ adolescência, o otimismo representa um importante fator de proteção frente a eventos estressores, atuando na diminuição de sintomas de depressão e promovendo uma maior competência social e emocional (Johnstone et al., 2014). Do mesmo modo, a autoestima é reconhecida como um importante recurso para o desenvolvimento positivo dos adolescentes, apresentando associação com maiores níveis de ajustamento e de bem- estar (Orth, Robins, Widaman, \& Conger, 2014). A satisfação com a vida, por sua vez, refere-se a uma avaliação global que o indivíduo tem em relação à sua vida e se relaciona negativamente com depressão, afetos negativos, pessimismo e outros indicadores de psicopatologia (Corrigan et al., 2013). Em adolescentes, a satisfação com a vida é um relevante indicador de desenvolvimento psicológico bem-sucedido (Saha, Huebner, Hills, Malone, \& Valois, 2014). Além disso, estudos apontam a satisfação com a vida como um preditor significativo do desenvolvimento de transtornos psiquiátricos, tais como depressão e ideação suicida em adolescentes (Horwitz, Berona, Czyz, Yeguez, \& King, 2016; Moksnes, \& Espnes, 2013; Saha, et. Al., 2014).

Diferentes autores vêm apontando para a importância de se investigar características psicológicas positivas em adolescentes, de modo a compreender o papel dos recursos pessoais e outros tipos de fatores de proteção no processo desenvolvimental (Albert, Chein, \& Steinberg, 2013; Alessandri, Eisenberg, \& Caprara, 2016; Caprara et al., 2014; Steiger, Allemand, Robins, \& Fend, 2014; Thomson, Schonert-Reichl, \& Oberle, 2015). Na literatura nacional, no entanto, estudos sobre os construtos da Psicologia Positiva em adolescentes ainda são escassos. Pureza et al. (2012) sugerem que tal escassez pode ser resultante da falta de instrumentos de medida validados e adequados para essa faixa etária. O presente estudo tem por objetivo apresentar evidências iniciais de validade da Escala de Positividade (EP) para uma amostra de adolescentes brasileiros.

\section{Método}

\section{Participantes}

Neste estudo foi utilizada uma amostra não probabilística de 638 adolescentes, sendo 432 do sexo feminino $(67,7 \%)$ e 206 do sexo masculino (32,3\%), com idades variando entre 11 e $19(M=15,19 ; D P=2,40)$. A maioria dos participantes é residente da região Sudeste (84,6\%), seguida pela região Sul $(9,2 \%)$, Nordeste $(2,5 \%)$, CentroOeste $(2,4 \%)$ e Norte $(1,3 \%)$. O contato com os participantes foi realizado principalmente por meio da internet (e-mail e redes sociais), de escolas de ensino médio e de contatos pessoais dos pesquisadores.

\section{Instrumentos}

Questionário Sociodemográfico. Instrumento composto por perguntas fechadas e que tem por objetivo obter informações que complementem os dados obtidos por meio dos questionários (ex.: características familiares, história de vida do adolescente, etc.).

Escala de Positividade (EP; Caprara et al., 2012a, 2012b). Instrumento unifatorial de autorrelato do tipo Likert composto por oito itens cujas respostas podem variar de 1 (discordo totalmente) a 5 (concordo totalmente). A versão brasileira foi traduzida e adaptada para o contexto brasileiro por Borsa et al. (Borsa et al., 2016; Borsa et al. 2015).

Escala de Esperança Disposicional para Adolescentes (ADHS, Snyder et al., 1997). Essa escala avalia o construto Esperança subdividido nos fatores Rota e Agenciamento. A ADHS foi desenvolvida para avaliar esperança como uma característica permanente e estável no tempo, o que indicaria temperamento, característica ou traço (Snyder et al., 1991). O instrumento original contém 12 itens, sendo quatro para a dimensão de rota, quatro para a dimensão agenciamento e quatro distratores. A versão original da escala apresentou boa consistência interna $(\alpha=0,71-0,84)$. A versão brasileira foi adaptada por Pacico et al. (2013) e apresentou estrutura unidimensional para o instrumento $(\alpha=0,80)$.

A Escala de Autoestima de Rosenberg - EAR (Rosenberg, 1989). Medida unidimensional constituída por dez afirmações que avaliam a autoestima global (sentimentos de autoestima e autoaceitação). Os itens são respondidos em uma escala do tipo Likert de quatro pontos, variando de concordo totalmente a discordo totalmente (Rosenberg, 1989). No Brasil, a escala foi validada por Hutz e Zanon (2011) e apresentou consistência interna alfa de Cronbach de 0,90.

Positive and Negative Affect Scale for Children (Panas-C8). Trata-se de uma versão reduzida da escala de Giacomoni e Hutz (2006), desenvolvida por Damásio, Pacico, Poletto e Koller (2012). A PANAS C8 avalia os afetos positivos (e. g., prazer, interesse, entusiasmo) e negativos (e. g., estados de humor aversivos, raiva, culpa, descontentamento e medo). Os itens são respondidos 
por meio de uma escala do tipo Likert de 5 pontos $(1-$ nem um pouco, 5 - muito). O estudo brasileiro da PANAS C8 contou com 1.160 participantes $(50,2 \%$ meninos) com média de idade de 11,18 anos $(D P=1,91)$. A análise fatorial exploratória apresentou claramente uma estrutura bifatorial, com cargas variando de 0,61 a 0,70 para Afetos Positivos e 0,56 a 0,76 para Afetos Negativos. O coeficiente alfa da escala foi de 0,77 para Afetos Positivos e 0,76 para Afetos Negativos.

Teste de Orientação de Vida Revisado (LOT-R). É um instrumento de autoavaliação para mensurar otimismo, desenvolvido por Scheier, Carver e Bridges (1994) e adaptado para adolescentes brasileiros por Bastianello, Pacico e Hutz (2014). O instrumento é composto por 10 itens, quatro deles distratores. Os sujeitos respondem às afirmações indicando seu grau de concordância a partir de uma escala de respostas do tipo Likert de 5 pontos (variando de discordo totalmente a concordo totalmente). Análises demonstraram estrutura unifatorial para escala, alfa de Cronbach $=0,77$.

Inventário de Saúde Mental (MHI-5; McHorney \& Ware, 1995). O MHI-5 é uma das oito sub-escalas que abrangem o SF-36 (Ware, Snow, Kosinski, \& Gandek, 1993). É composto por cinco itens, respondidos em escala tipo Likert, que avaliam sintomas de depressão e ansiedade. Escores mais altos indicam melhores níveis de saúde mental. A versão brasileira foi validada por Damásio, Borsa e Koller (2014) apresentando adequados índices de ajuste $(\mathrm{CFI}=0,99$; $\mathrm{TLI}=0,99$; RMSEA $(90 \% \mathrm{CI})=0,07$ $(0,05-11)$; SRMR $=0,02)$.

\section{Procedimentos Éticos e de Coleta de Dados}

A amostra total desse estudo foi constituída de três subamostras, coletadas em três estudos distintos, os quais foram aprovados pelo Comitê de Ética em Pesquisa da Universidade Federal do Rio de Janeiro (UFRJ) e pela Comissão de Ética da Pontifícia Universidade Católica do Rio de Janeiro (PUC-Rio).

Para a coleta presencial com os adolescentes com idade inferior a 18 anos, foram realizados contatos pessoais ou por telefone com as escolas para a realização da coleta de dados. O Termo de Consentimento Livre e Esclarecido (TCLE) foi entregue aos pais, mães e responsáveis em duas vias (uma para os pais e outra para os pesquisadores), por meio da escola, e foram devolvidos aos pesquisadores devidamente preenchidos e assinados. A privacidade dos participantes, o sigilo e a confidencialidade dos dados foram garantidas. A coleta de dados presencial só aconteceu mediante a concordância e o consentimento dos pais ou responsáveis, bem como do posterior assentimento dos adolescentes. A aplicação dos questionários ocorreu de forma coletiva, na sala de aula, ou em espaço cedido pelas escolas, e levou em média de 20 a 40 minutos.

A coleta de dados on-line, por sua vez, foi realizada apenas com participantes que tinham, na data da pesquisa, 18 anos completos ou mais. Para tal, foi utilizada a ferramenta SurveyMonkey ${ }^{\circledR}$, que consiste em um software, em formato de website, que tem por objetivo possibilitar a coleta de dados por meio de questionários on-line. Os participantes foram contatados via e-mails previamente fornecidos voluntariamente aos pesquisadores e por meio da técnica "bola de neve" (Patton, 1990). Na coleta de dados on-line, o TCLE estava na primeira página da pesquisa e o questionário só era iniciado caso o participante marcasse a opção de concordância com o termo. A coleta de dados só ocorreu mediante a concordância dos participantes, que poderiam desistir da pesquisa a qualquer momento da aplicação.

\section{Procedimentos de Análise dos Dados}

Para avaliar as evidências de validade baseada na estrutura interna do instrumento, a amostra total foi aleatoriamente dividida em duas partes. Realizou-se uma análise fatorial exploratória (EFA) com a primeira metade da amostra $(n=319)$. Considerando o nível de medida ordinal das variáveis e da violação da hipótese e da normalidade multivariada dos dados, a análise foi conduzida baseando-se numa matriz de correlação policórica (Holgado-Tello, Chacón-Moscoso, BarberoGarcia, \& Vila-Abad, 2010) com o método de extração da Minimum Rank Factor Analysis (MRFA) (Shapiro \& ten Berge, 2002). O MRFA minimiza a variância comum residual no processo de extração do fator e possibilita a interpretação da proporção de variância comum que é explicada pelos fatores retidos (Lorenzo-Seva \& Ferrando, 2006). O critério de retenção de fator foi o método Hull (HM, Lorenzo-Seva, Timmerman, \& Kiers, 2011). Até o presente momento, o HM demonstrou ser o método de retenção de fator mais confiável (Lorenzo-Seva et al., 2011). A confiabilidade da escala foi avaliada utilizando coeficiente alfa (Cronbach, 1951). O programa utilizado para rodar a AFE foi o Factor Version 9.3 (Lorenzo-Seva \& Ferrando, 2006).

Uma análise fatorial confirmatória (CFA), utilizando o método Weighted Least Squares Means and Varianceadjusted (WLSMV; Muthén \& Muthén, 2012) adequado para variáveis ordinais com desvios de normalidade, foi conduzida com a segunda metade da amostra $(n=319)$, para validade cruzada da estrutura obtida na análise fatorial exploratória. Os índices de ajuste utilizados foram: Índice de ajuste comparativo (CFI), índice Tucker-Lewis (TLI) e raiz quadrada média do erro de aproximação (RMSEA). O modelo é aceito quando os valores de CFI e TLI são acima de 0,90 (preferencialmente acima de $0,95)$, e os valores de RMSEA abaixo de 0,06 para indicar ajuste adequado (com $90 \%$ do seu intervalo de confiança não maior que 0,10$)$ (Brown, 2006).

Foram realizadas correlações de Pearson para verificar evidências de validade baseada em variáveis externas, especificamente no que se refere aos padrões de convergência entre a EP e a ADHS, EAR, LOT-R e MHI-5. 
Esses instrumentos foram respondidos por uma subamostra composta de 398 participantes, sendo 275 do sexo feminino $(69,1 \%)$ e 123 do sexo masculino (30,9\%), com idades variando entre 11 e $19(M=13,79 ; D P=1,84)$. Foram esperadas correlações positivas moderadas a altas entre a EP e as demais variáveis.

Correlações de Pearson também avaliaram a relação entre a EP e idade. Teste $t$ de Student avaliou possíveis diferenças entre os níveis de positividade entre homens e mulheres. Para todas as análises, foram implementados procedimentos de reamostragem (bootstrapping; 1000 reamostragens, com intervalo de confiança 99\%), com vistas a apresentar maior confiabilidade aos resultados, corrigir possíveis desvio de normalidade da amostra e apresentar intervalo de confiança de $99 \%$ para as diferenças de média (Chernick, \& LaBudde, 2014). O tamanho de efeito do teste $t$ foi calculado por meio do $d$ de Cohen (Cohen, 1988).

\section{Resultados}

\section{Análise Fatorial Exploratória (AFE)}

O método Hull sugeriu um fator como o mais representativo para os dados. A solução unifatorial foi responsável por $68,94 \%$ da variância explicada. Além disso, todos os itens carregaram satisfatoriamente no fator (cargas fatoriais $\geq 0,40$; Tabela 3) exceto o item 4 ("Algumas vezes, o futuro parece incerto para mim") (carga fatorial $\leq 0,40$; Tabela 1).

Tabela 1

Análise Fatorial Exploratória da Escala de Positividade para Adolescentes (EP)

\begin{tabular}{lc}
\hline & Fator \\
\cline { 2 - 2 } & EP \\
\hline Item 8 & $0,809^{*}$ \\
Item 6 & $0,708^{*}$ \\
Item 7 & $0,684^{*}$ \\
Item 5 & $0,669^{*}$ \\
Item 1 & $0,658^{*}$ \\
Item 3 & $0,617^{*}$ \\
Item 2 & $0,455^{*}$ \\
Item 4 & $-0,266$ \\
\hline Eigenvalue & 3,16 \\
Variância Explicada & $68,94 \%$ \\
Alpha de Cronbach & 0,73 \\
\hline
\end{tabular}

Nota. ${ }^{*}=$ itens que carregaram satisfatoriamente no fator (carga fatorial $\geq 0,40$ ). Valores sem asterisco = itens que não carregaram (carga fatorial $\leq 0,40$ )

\section{Análise Fatorial Confirmatória}

A AFC foi aplicada na segunda metade da amostra $(n=319)$ para validação cruzada do modelo obtido na análise fatorial exploratória. A estrutura encontrada para a solução unifatorial apresentou os seguintes índices de ajuste $\chi^{2}(d f)=89,784$ (14), $p<0,001 ; \mathrm{CFI}=0,94$; TLI=0,91; RMSEA=0,13 (90\% IC: $0,11-0,16)$.

Embora a adequação tenha sido aceitável para a maioria dos indicadores, o valor de RMSEA foi maior do que o aceitável (Brown, 2006). A fim de compreender esse resultado, uma análise dos índices de modificação (IM) revelou que dois itens aumentaram significativamente o valor do qui-quadrado, consequentemente aumentando o valor do RMSEA. As correlações de erro foram: Erro 6 ("Às vezes, o futuro me parece incerto") e Erro 1 ("Eu tenho grande fé no futuro"), IM=69.242. Quando consideradas essas modificações, o modelo apresentou índices ideais de ajuste: $\chi^{2}(d f)=20,61$ (13), $\quad p=0,08 ; \quad$ TLI $=0,99 ; \quad$ CFI $=0,99 ; \quad$ RMSEA $=0,04$ $(0,00-0,076)$. Esses resultados se referem ao modelo sem o item 4, conforme a solução encontrada pela AFE. Para fins de verificação, também foi testado o modelo ajustado com a presença do item 4, que acabou apresentando índices de ajustes levemente melhores do que com a exclusão do item: $\chi^{2}(d f)=26,151(19), p=0,13$; TLI=0,99; $\mathrm{CFI}=0,99$; RMSEA $=0,03(0,00-0,064)$. Considerando os resultados contraditórios entre a EFA, que sugere a exclusão do item 4, e da CFA, que apresenta índices de ajuste um pouco mais adequados considerando a presença do item 4, optou-se por avaliar a validade convergente da escala com os demais indicadores, utilizando a versão completa do instrumento.

\section{Validade Convergente}

A validade convergente foi conduzida empregando a correlação entre a EP e demais medidas correlatas e constitutivas. Como esperado, a EP correlacionou-se positivamente com o tamanho de efeito alto com a LOT-R, com a PANAS-AP, com a EAR, PANAS-C8 e com a ADSH. Além disso, também conforme era esperado, a EP se correlacionou negativamente com a MHI-5 e com a PANAS-AN, conforme demonstrado na Tabela 2.

Tabela 2

Correlações entre a EP e as demais Escalas Convergentes

\begin{tabular}{lc}
\hline \multirow{2}{*}{ Escalas } & Correlações \\
\cline { 2 - 2 } & EP \\
\hline MHI-5 & $0,55^{* *}$ \\
LOT-R & $0,53^{* *}$ \\
EAR & $0,58^{* *}$ \\
ADHS & $0,58^{* *}$ \\
PANAS-C8-AP & $0,50^{* *}$ \\
PANAS-C8-AN & $-0,33^{* *}$ \\
\hline
\end{tabular}

Nota. ${ }^{* *}=p<0,01 ;$ MHI-5 - Índice de saúde mental; LOT-R Escala de Otimismo; EAR - Escala de Autoestima de Rosenberg; PANAS-C8-AP - Escala de Afetos Positivos e Negativos para Crianças (Afetos positivos); PANAS-C8-AN - Escala de Afetos Positivos e Negativos para Crianças (Afetos negativos) 


\section{Positividade e Relações com Variáveis Sociodemográficas}

Foi encontrada uma correlação negativa e significativa entre os índices de positividade e idade $[r=-0,134$; $p<0,01]$. O teste $\mathrm{t}$ de Student apontou diferença estatisticamente significativa nos escores da EP entre meninos e meninas $[t(636)=-2,43 ; p<0,05 ; d=0,21]$. Meninos apresentaram escores de positividade superiores às meninas, porém o tamanho de efeito da diferença foi pequeno.

\section{Discussão}

As análises fatoriais exploratória e confirmatória indicaram solução unifatorial para a Escala de Positividade (EP), resultado esperado e coerente com a estrutura original da escala (Caprara et al., 2012a) e sua versão brasileira para adultos (Borsa et al., 2015). Na AFC, foi apresentada elevada correlação residual entre os itens 1 ("Eu tenho muita confiança no futuro") e 6 ("Eu vejo o futuro com esperança e entusiasmo"), o que sugere a existência de uma possível sobreposição de conteúdo entre esses itens, possivelmente devido aos dois itens expressarem uma visão positiva e otimista em relação ao futuro. Tal resultado corrobora diversos outros estudos que avaliaram as propriedades psicométricas da EP, sugerindo um padrão consistente de correlação residual entre esses dois indicadores (e.g., Borsa et al., 2015, 2016; Caprara et al., 2010, 2012a, 2012b).

O item 4 ("Algumas vezes, o futuro parece incerto para mim"), diferentemente dos resultados da aplicação em adultos (Borsa et al., 2015), não apresentou carga fatorial adequada na amostra do presente estudo. Uma explicação teórica plausível para esse resultado pode residir no fato de que os adolescentes possivelmente têm uma dificuldade maior do que os adultos para lidar com o futuro, por serem mais instáveis e possuírem uma menor capacidade de planejamento a longo prazo (Albert, Chein, \& Steinberg, 2013). Isso ocorreria como uma consequência do fato de que esses níveis de planejamento aumentam somente conforme a idade avança, em decorrência da maturação de regiões cerebrais relacionadas ao controle da impulsividade e planejamento (Albert, Chein, \& Steinberg, 2013). Sendo assim, a incerteza em relação ao futuro pode ser mais comum e normal em adolescentes, não se caracterizando necessariamente como algo negativo.

Entretanto, como existem outros itens na escala que também são relacionados ao futuro e obtiveram carga fatorial aceitável, é possível que a própria formulação do item 4 tenha prejudicado seus escores. Ressalta-se aqui o fato de que ele é o único item negativo da escala, o que pode confundir ou gerar algum tipo de viés no respondente, além de possuir o indicador de frequência "algumas vezes" que pode dificultar as respostas por meio da escala do tipo Likert. Além disso, o termo "incerto" pode se comportar de maneira ambivalente e acabar neutralizando o item, pois enxergar o futuro como algo "incerto" não necessariamente é algo ruim, uma vez que a incerteza pode se referir tanto a um desfecho negativo quanto positivo em relação a como será o futuro.

Ainda em relação ao item 4 , nota-se que os índices de ajustes obtidos por meio da AFC tendem a melhorar quando se mantém o item na escala. Apesar de ser contraditório em relação à baixa carga fatorial desse item, conforme apresentado na EFA, esse resultado mostra que, não necessariamente, o modelo piora com a presença do item. Com base nos resultados da AFC, os modelos com e sem o item acabam sendo muito similares. É importante ressaltar que tanto no estudo original de construção e validação dessa escala (Caprara et al., 2012a) quanto no estudo realizado no Brasil que apresentou as propriedades psicométricas da versão brasileira da EP (Borsa et al., 2015), nota-se que o item 4 também apresentou uma carga fatorial abaixo de 0,40 . É evidente, portanto, a necessidade de se discutir a pertinência desse item para a EP em estudos posteriores. Diante dessas implicações, neste estudo optou-se por manter o item 4, ainda que sua carga fatorial tenha sido baixa, até que novos estudos sejam realizados em amostras mais amplas e diversificadas, trazendo novas evidências para a sua manutenção ou exclusão.

A validade convergente da escala foi evidenciada por meio de correlações positivas moderadas entre a EP e os indicadores de esperança, otimismo, autoestima, e saúde mental. Esse resultado era teoricamente esperado, uma vez que se tratam de variáveis correlatas à positividade, conforme indicam outros estudos (Borsa et al., 2016; Caprara et al., 2012a; Caprara, Eisenberg, \& Alessandri, 2016). Pessoas com altos índices de positividade tendem a ter maiores índices de esperança, a serem mais otimistas, a apresentarem maior autoestima e ter maiores escores de saúde mental (especificamente, menores índices de depressão e ansiedade).

Em relação às variáveis sociodemográficas, foi encontrada uma correlação negativa e fraca entre idade e os escores da EP, indicando que, dentro do período da adolescência, o efeito da idade nos escores de positividade é pouco importante. Esse resultado está de acordo com estudos que demonstram que a positividade tende a ser uma característica relativamente estável no indivíduo ao longo do tempo (Alessandri, Caprara, \& Tisak, 2012). Em relação ao sexo, meninos apresentaram escores de positividade superiores às meninas, mas essa diferença teve tamanho de efeito pequeno. No estudo original da EP com adultos, não foram encontradas diferenças entre homens e mulheres (Caprara et al., 2012a). Assim, futuros estudos são necessários para esclarecer se há diferenças reais nos níveis de positividade entre homens e mulheres.

\section{Considerações Finais}

O presente estudo tem por objetivo apresentar evidências iniciais de validade da Escala de Positividade 
(EP) para uma amostra de adolescentes. Esse é o primeiro estudo brasileiro dedicado a levantar evidências de validade de uma medida de positividade para adolescentes. As propriedades psicométricas dessa escala se mostraram adequadas para esse fim, indicando evidências favoráveis à utilização da EP para mensurar a positividade em adolescentes. Os resultados encontrados também corroboraram os resultados de estudos internacionais (Caprara et al., 2012a) e brasileiros da escala (Borsa et al., 2015; Borsa et al., 2016).

É sabido que, na adolescência, características pessoais como competência social, capacidade senso de identidade, autoestima positiva, confiança no futuro, autoeficácia, dentre outras, são protetoras do desenvolvimento (Milioni, Alessandri, Eisenberg, \& Caprara, 2016; Albert, Chein, \& Steinberg, 2013). A positividade é um construto que tem entre as suas variáveis constituintes a autoestima e a satisfação com a vida (Caprara et al., 2010), as quais apresentam forte relação com otimismo e com a capacidade de lidar com sucesso diante dos eventos estressores (Milioni et al., 2016). Assim, o entendimento desse construto se faz necessário de maneira a permitir que sejam pensadas práticas que estimulem essas características como fatores protetivos no desenvolvimento de adolescentes.

Além disso, este estudo é uma contribuição para a literatura brasileira relacionada à Psicologia Positiva, uma vez que contribui para a investigação empírica e científica de aspectos positivos do desenvolvimento humano. Tendo em vista a necessidade que a Psicologia Positiva tem de entender como os fatores positivos contribuem para a saúde física e subjetiva e para o funcionamento saudável de grupos e instituições e considerando o número escasso de instrumentos válidos para mensurar esses aspectos em adolescentes, estudos como este se mostram como importantes contribuições.

No que diz respeito às limitações do estudo, destaca-se a restrição geográfica da amostra, proveniente em sua maioria da região Sudeste. Sugere-se que novos estudos sejam conduzidos utilizando amostras mais amplas e de diferentes regiões do Brasil. Também se sugere estudos com grupos clínicos e não clínicos e que permitam investigar novas evidências baseadas em variáveis externas. Além disso, é necessária a realização de novos estudos com o intuito de verificar e levantar novas evidências sobre a pertinência do item 4 da EP para essa população. Estudos que relacionem a positividade com outras variáveis sociodemográficas e de personalidade também podem ser esclarecedores, bem como a realização de estudos longitudinais - que podem trazer boas contribuições em relação ao entendimento do desenvolvimento e estabilidade das características positivas nos adolescentes.

\section{Referências}

Albert, D., Chein, J., \& Steinberg, L. (2013). The teenage brain: Peer influences on adolescent decision making. Current directions in psychological science, 22(2), 114-120.

Alessandri, G., Caprara, G. V., \& Tisak, J. (2012). The unique contribution of positive orientation to optimal functioning. European Psychologist, 17(1), 44-54. doi: 10.1027/1016-9040/a000070

Alessandri, G., Vecchione, M., Tisak, J., DeIana, G., Caria, S., \& Caprara, G. V. (2012). The utility of positive orientation in predicting job performance and organizational citizenship behaviors. Applied Psychology, 61(4), 669-698. doi: 10.1111/j.1464-0597.2012.00511.x

Bastianello, M. R., Pacico, J. C., \& Hutz, C. S. (2014). Optimism, self-esteem and personality: Adaptation and validation of the Brazilian Version of the Revised Life Orientation Test (LOT-R). Psico-USF, 19(3), 523-531. doi: 10.1590/1413-827120140190030

Borsa, J. C., Damásio, B. F., \& Koller, S. H. (2016). Positivity scale (POS): New evidence of validity in the brazilian context. Psico-USF, 21(1), 1-12. doi: 10.1590/1413-82712016210101

Borsa, J. C., Damásio, B. F., Souza, D. S. D., Koller, S. H., \& Caprara, G. V. (2015). Psychometric properties of the positivity scale-Brazilian version. Psicologia: Reflexão e Crítica, 28(1), 61-67. doi: 10.1590/1678-7153.201528107

Brown, T. A. (2006). Confirmatory factor analysis for applied research. New York: The Guilford Press.

Caprara, G. V., Alessandri, G., Eisenberg, N., Kupfer, A., Steca, P., Caprara, M. G., \& Abela, J. (2012a). The Positivity Scale. Psychological Assessment, 24(3), 701-712. doi:10.1037/a0026681

Caprara, G. V., Alessandri, G., Trommsdorff, G., Heikamp, T., Yamaguchi, S., \& Suzuki, F. (2012b). Positive orientation across three cultures. Journal of Cross-Cultural Psychology, 43(1), 77-83. doi: 10.1177/0022022111422257

Caprara, G. V., Eisenberg, N., \& Alessandri, G. (2016). Positivity: The dispositional basis of happiness. Journal of Happiness Studies, 1-19. doi: 10.1007/s10902-016-9728-y

Caprara, G. V., Kanacri, B. P. L., Gerbino, M., Zuffianò, A., Alessandri, G., Vecchio, G., ... \& Bridglall, B. (2014). Positive effects of promoting prosocial behavior in early adolescence: Evidence from a school-based intervention. International Journal of Behavioral Development, 38(4), 386-396. doi: 10.1177/0165025414531464

Caprara, G. V., Steca, P., Alessandri, G., Abela, J. R. Z., \& McWhinnie, C. M. (2010). Positive orientation: Explorations on what is common to life satisfaction, self-esteem, and optimism. Epidemiologia e Psichiatria Sociale, 19(1), 63-71. doi: 10.1017/S1121189X00001615

Chernick, M. R., \& LaBudde, R. A. (2014). An introduction to bootstrap methods with applications to R. John Wiley \& Sons.

Cohen, J. (1988). Statistical power analysis for the behavioral sciences. New Jersey: Lawrence Erlbaum.

Corrigan, J. D., Kolakowsky-Hayner, S., Wright, J., Bellon, K., \& Carufel, P. (2013). The satisfaction with life scale. The Journal of head trauma rehabilitation, 28(6), 489-491. doi: 10.1097/HTR.0000000000000004 
Costantini, G., Perugini, M., Dentale, F., Barbaranelli, C., Alessandri, G., Vecchione, M., \& Caprara, G. V. (2016). Assessing positive orientation with the implicit association test. European Journal of Psychological Assessment. doi: 10.1027/1015-5759/a000362

Cronbach, L. J. (1951). Coefficient alpha and the internal structure of tests. Psychometrika, 16(3), 297-334. doi: 10.1007/BF02310555

Csikszentmihalyi, M. (2014). Toward a psychology of optimal experience. Springer Netherlands.

Damásio, B. F., Borsa, J. C., \& Koller, S. H. (2014). Adaptation and psychometric properties of the Brazilian version of the Five-item Mental Health Index (MHI-5). Psicologia: Reflexão e Crítica, 27(2), 323-330. doi: 10.1590/1678-7153.201427213

Damásio, B. F., Pacico, J. C., Poletto, M., \& Koller, S. H. (2012). Refinement and psychometric properties of the Eight-Item Brazilian Positive and Negative Affective Schedule for Children (PANAS-C8). Journal of Happiness Studies, 14(4), 1363-1378. doi: 10.1007/ s10902-012-9383-x

Diener, E., Kanazawa, S., Suh, E. M., \& Oishi, S. (2015). Why people are in a generally good mood. Personality and Social Psychology Review, 19(3), 235-256. doi: 10.1177/1088868314544467

Diener, E., Scollon, C. K. N., Oishi, S., Dzokoto, V., \& Suh, E. M. (2000). Positivity and the construction of life satisfaction judgments: Global happiness is not the sum of its part. Journal of Happiness Studies, 1(2), 159-176. doi: 10.1017/S1121189X00001615

Giacomoni, C. H., \& Hutz, C. S. (2006). Escala de afeto positivo e negativo para crianças: Estudo de construção e validação (Positive and Negative Affect Scale for Children: Construction and validation study). Revista Semestral da Associação Brasileira de Psicologia Escolar e Educacional (ABRAPEE), 10(2), 235-245.

Holgado-Tello, F., Chacón-Moscoso, S., Barbero-García, I., \& Vila-Abad, E. (2010). Polychoric versus Pearson correlations in exploratory and confirmatory factor analysis of ordinal variables. Quality and Quantity, 44(1), 153-166. doi: 10.1007/s11135-008-9190-y

Horwitz, A. G., Berona, J., Czyz, E. K., Yeguez, C. E., \& King, C. A. (2016). Positive and negative expectations of hopelessness as longitudinal predictors of depression, suicidal ideation, and suicidal behavior in high-risk adolescents. Suicide and life-threatening behavior. doi: 10.1111/sltb.12273

Hutz, C., \& Zanon, C. (2011). Revisão da adaptação, validação e normatização da escala de autoestima de Rosenberg. Avaliação Psicológica, 10(1), pp. 41-49.

Johnstone, J., Rooney, R. M., Hassan, S., \& Kane, R. T. (2014). Prevention of depression and anxiety symptoms in adolescents: 42 and 54 months follow-up of the Aussie Optimism Program-Positive Thinking Skills. Frontiers in psychology, 5, 364.

Lorenzo-Seva, U., \& Ferrando, P. J. (2006). FACTOR: A computer program to fit the exploratory factor analysis model. Behavioral Research Methods, Instruments and Computers, 38(1), 88-91. doi: 10.3758/BF03192753

Lorenzo-Seva, U., \& Ferrando, P. J. (2006). Factor (9.2) [Programa de computador].

Lorenzo-Seva, U., Timmerman, M. E., \& Kiers, H. A. L. (2011). The Hull Method for selecting the number of common factors. Multivariate Behavioral Research, 46(2), 340-364. doi: 10.1080/00273171.2011.564527

Lyubomirsky, S., \& Layous, K. (2013). How do simple positive activities increase well-being?. Current Directions in Psychological Science, 22(1), 57-62. doi: 10.1177/0963721412469809

McHorney, C. A., \& Ware, J. E., Jr. (1995). Construction and validation of an alternate form general mental health scale for the Medical Outcomes Study Short-Form 36-Item Health Survey. Medical Care, 33(1), 15-28.

Milioni, M., Alessandri, G., Eisenberg, N., \& Caprara, G. V. (2016). The role of positivity as predictor of ego-resiliency from adolescence to young adulthood. Personality and Individual Differences, 101(1), 306-311.

Moksnes, U. K., \& Espnes, G. A. (2013). Self-esteem and life satisfaction in adolescentes-gender and age as potential moderators. Quality of Life Research, 22(10), 2921-2928. doi: 10.1007/s11136-013-0427-4

Muthén, L. K., \& Muthén, B. O. (2012). Mplus: Statistical analysis with latent variables. User's guide. Los Angeles: Muthén \& Muthén.

Oles, P. K., Alessandri, G., Oles, M., Bak, W., Jankowski, T., Laguna, M., \& Caprara, G. V. (2013). Positive orientation and generalized selfefficacy. Studia Psychologica, 55(1), 47.

Orejudo-Hernández, S., Aparicio-Moreno, L., \& Cano-Escoriaza, J. (2013). Personal competencies of Spanish students pursuing different academic careers. Contributions and reflections from Positive Psychology. Journal of Behavior, Health E Social Issues, 5(2), 63-78. doi: 10.5460/jbhsi.v5.2.42253

Orth, U., Robins, R. W., Widaman, K. F., \& Conger, R. D. (2014). Is low self-esteem a risk factor for depression? Findings from a longitudinal study of Mexican-origin youth. Developmental psychology, 50(2), 622. doi: 10.1037/a0033817

Pacico, J. C., Bastianello, M. R., Zanon, C., \& Hutz, C. S. (2013). Adaptation and validation of the dispositional hope scale for adolescents. Psicologia: Reflexão e Crítica, 26(3), 488-492.

Patton, M. Q. (1990). Qualitative evaluation and research methods (2nd ed.). Newbury Park, CA: Sage.

Pureza, J. R., Kuhn, C. H. C., Castro, E. K., \& Lisboa, C. S. M. (2012). Psicologia positiva no Brasil: Uma revisão sistemática da literatura. Revista Brasileira de Terapias Cognitivas, 8(2), 109-117.

Rosenberg, M. (1989). Society and the adolescent self-image. Revised edition. Middletown, CT: Wesleyan University Press.

Saha, R., Huebner, E. S., Hills, K. J., Malone, P. S., \& Valois, R. F. (2014). Social coping and life satisfaction in adolescents. Social Indicators Research, 115(1), 241-252. doi: 10.1007/s11205-012-0217-3

Santrock, J. W. (2014). Adolescência. (14 ed.). Porto Alegre: Artmed.

Scheier, M. F., Carver, C. S. \& Bridges, M. W. (1994). Distinguishing Optimism from neuroticism (and trait anxiety, self- mastery, and self- esteem): A reevaluation of the life orientation test. Journal of Personality And Social Psychology, 67(6), 1063-1078. doi: 10.1037/0022-3514.67.6.1063

Seligman, M. E., \& Csikszentmihalyi, M. (2014). Positive psychology: An introduction. Springer Netherlands.

Senna, S. R. C. M., \& Dessen, M. A. (2012). Contribuições das teorias do desenvolvimento humano para a concepção contemporânea da adolescência. Psicologia: Teoria e Pesquisa, 28(1), 101-108.

Shapiro, A., \& ten Berge, J. M. F. (2002). Statistical inference of minimum rank factor analysis. Psychometrika, 67(1), 79-94. doi: 10.1007/ BF02294710

Snyder, C. R., Harris, C., Anderson, J. R., Holleran, S. A., Ir- ving, L. M., Sigmon, S. T., Harney, P. (1991). The will and the ways: Development and validation of an individual-differences measure of hope. Journal of Personality and Social Psychology, 60(4), 570-585. doi: 10.1037/0022-3514.60.4.570

Steiger, A. E., Allemand, M., Robins, R. W., \& Fend, H. A. (2014). Low and decreasing self-esteem during adolescence predict adult depression two decades later. Journal of personality and social psychology, 106(2), 325. 
Thomson, K. C., Schonert-Reichl, K. A., \& Oberle, E. (2015). Optimism in early adolescence: Relations to individual characteristics and ecological assets in families, schools, and neighborhoods. Journal of Happiness Studies, 16(4), 889-913. doi: 10.1007/s10902-014-9539-y

Ware, J. E., Snow, K. K., Kosinski, M., \& Gandek, B. (1993). SF-36 Health Survey manual and interpretation guide. Boston: The Health Institute, New England Medical Center.

World Health Organization (WHO, 2014). First WHO report on suicide prevention. Recuperado de http://www.who.int/mediacentre/news/ releases/2014/suicide-prevention-report/en/

\section{Sobre os autores}

Juliane Callegaro Borsa é professora adjunta do Departamento de Psicologia e do Programa de Pós-Graduação em Psicologia Clínica da Pontifícia Universidade Católica do Rio de Janeiro (PUC-Rio), fundadora e coordenadora do Grupo de Pesquisa APlab - Pessoas \& Contextos, coordenadora do Laboratório de Avaliação Psicológica de Crianças e Adolescentes (LAPcriad), mestre em Psicologia Clínica pela Pontifícia Universidade Católica do Rio Grande do Sul (PUCRS) e doutora em Psicologia pela Universidade Federal do Rio Grande do Sul (UFRGS). Áreas de interesse: avaliação psicológica; construção, adaptação e validação de instrumentos psicológicos; avaliação de problemas emocionais e comportamentais na infância; desenho da figura humana; avaliação de indicadores de saúde mental ao longo do ciclo vital.

Andréa Jannotti Nogueira Rodrigues é psicóloga formada pela Universidade Federal do Rio de Janeiro (UFRJ), mestre em Psicologia Clínica da Pontifícia Universidade Católica do Rio de Janeiro (PUC-Rio) e foi bolsista CAPES de mestrado no Programa de Pós-Graduação da PUC-Rio. Atualmente faz o doutoramento em Neuropsicologia na Faculdade de Psicologia e Ciência da Educação da Universidade do Porto. É professora da graduação e pós-graduação da UNESA e psicóloga clínica. Dedica-se a estudos relacionados à neuropsicologia do desenvolvimento. Tem interesse em avaliação psicológica, construção, adaptação e validação de instrumentos; psicologia baseada em evidências; neuropsicologia do desenvolvimento; intervenção; infância; adolescência; psicologia escolar.

Bheatrix Bienemann é psicóloga formada pela Universidade Federal do Rio de Janeiro (UFRJ) e mestra em Psicologia Clínica pela Pontifícia Universidade Católica do Rio de Janeiro (PUC-Rio), tendo recebido bolsa CNPq durante o mestrado. Atualmente é bolsista de doutorado CNPq no Programa de Pós-Graduação em Psicologia Clínica da PUC-Rio. Dedica-se a estudos relacionados à neurociência, dependência química e uso de substâncias. Também tem interesse em construção, adaptação e validação de instrumentos psicométricos; psicologia baseada em evidências, epistemologia e metodologia de pesquisa em Psicologia.

Bruno Figueiredo Damásio é professor adjunto do Instituto de Psicologia (IP) e do Programa de Pós-Graduação em Psicologia da Universidade Federal do Rio de Janeiro (UFRJ), chefe do Departamento de Psicometria do IP (2015-), coordenador do Laboratório de Psicometria e Psicologia Positiva (LP3). Tem como foco de pesquisa a Psicometria e a Psicologia Positiva e dedica-se aos estudos de construção, adaptação e validação de instrumentos psicológicos, utilizando Modelagem por Equações Estruturais e Teoria de Resposta ao Item. Tem interesse nos seguintes temas: sentido de vida, bem-estar, resiliência, recursos psicossociais, pesquisas transculturais e longitudinais. 